\title{
Dually Cross-linked Supramolecular Hydrogel for Cancer Biomarker Sensing
}

Jie $\mathrm{Li}^{1+}$, Chendong $\mathrm{Ji}^{2+}$, Baozhong Lü ${ }^{2}$, Maksim Rodin${ }^{1}$, Jan Paradies ${ }^{1}$, Meizhen Yin ${ }^{2} *$, Dirk Kuckling $^{1 *}$

1 Department of Chemistry, Paderborn Universitry, Warburgerstraße 100, D-33098 Paderborn, Germany

2 State Key Laboratory of Chemical Resource Engineering, Beijing Advanced Innovation Center for Soft Matter Science and Engineering, Beijing Laboratory of Biomedical Materials, Beijing University of Chemical Technology, Beijing 100029, China

\section{Reagents and Instruments}

$N, N$-Dimethylacrylamide ( $>99.0 \%, \mathrm{TCI})$ was vacuum distillation before used. The following chemicals were purchased from commercial sources and used as received. dimethymaleic anhydride (97\%, Acros Organics), 1,2-Diaminoethane (98\%, Acros Organics), di-tert-butyl-dicarbonate (99\%, Acros Organics). 1-Hydroxybenzotriazole hydrate (HOBt) (>97.0\%, Sigma Aldrich), $N-(3-$ Dimethylaminopropyl)- $N$ '-ethylcarbodiimide hydrochloride (EDC) (>98.0\%, Sigma Aldrich), acryloyl chloride (96\%, Alfa Aesar), allylamine (98+ \%, Alfa Aesar), tosylchloride (98\%, Alfa Aesar), ferrocene carboxylic acid (98\%, Alfa Aesar), N,N-Diisopropylethylamine (97\%, Alfa Aesar), 2-Methylalanine (>98.0\%, TCI), 2-Aminoethanethiol hydrochloride $(>95.0 \%$, TCI), trifluoroacetic acid ( $>99.0 \%$, TCI), 2,6-Di-tert-butyl-p-cresol (BHT) (> $99 \%$, Fluka), ethyl chloroformate (99\%, Fluka), thioacetic acid (>97\%, Fluka), Oleoyl-L- $\alpha$-lysophosphatidic acid sodium salt ( $\geq 98 \%$, Chem Cruz), L- $\alpha$-Lysophosphatidylcholine (from egg yolk) (Chem Cruz), DGlycerate 3-phosphate disodium salt (Chem Cruz). All other normal chemicals and solvents were of analytical grade and were used without further purification.

${ }^{1} \mathrm{H}$ and ${ }^{13} \mathrm{C}$ NMR spectra were recorded on a Bruker AV-500 spectrometer $\left(500 \mathrm{MHz}\right.$ for ${ }^{1} \mathrm{H}$ and $125 \mathrm{MHz}$ for $\left.{ }^{13} \mathrm{C}\right)$, with chemical shifts (d) reported in ppm relative to solvent peak (7.26 ppm for $\mathrm{CDCl}_{3}$ and $2.50 \mathrm{ppm}$ for DMSO) standards and coupling constants $(J)$ reported in Hz. Highresolution mass spectra (HRMS) were recorded by electrospray ionization (ESI) on a Waters SYNAPT'т G2 HDMS'м mass spectra. The molar masses and molar mass distribution (Mw/Mn) 
were performed on a gel permeation chromatography (GPC) at a flow rate of $1 \mathrm{~mL} / \mathrm{min}$ on a Merck Hitachi 655A-11 Liquid Chromatograph connected to a Knauer Smartline RI Detector 2300 and a Merck Hitachi L-4200 UVVIS Detector. The instrument was equipped with a PSS-GRAM $10^{3} \AA$ and PSS-GRAM $10^{2} \AA$ column and all samples were calibrated by poly(methylmethacrylate) standards. Supramolecular polymer size was carried out on a dynamic light scattering (DLS) apparatus of Malvern Nano-ZS at an angle of incidence of $15^{\circ}$. Photo crosslinking process was performed by an OmniCure ${ }^{\circledR}$ S1500 spot UV curing lamp with a unique and patented 200 Watt Intelli-Lamp.

All structures were preoptimized with the program gfn-xtb Version 2.0[1] using the implemented gbsa solvent model for water. The structures were then subjected to DFT based calculations using the ORCA 4.0.1 quantum chemical package.[2] The molecules were optimized at the PBEh-3c / def2-mSVP [3] level of theory using density fitting (RIJCOSX) and D3BJ dispersion correction.[4] Vibrational properties were calculated at the same level of theory. The final energies were computed at the $\omega$ B97-D3/def2-TZVP[5] level of theory with density fitting. Solvent correction to the final energies acquired from gfn-XTB gbsa solvent model.

\section{Figures and Tables}
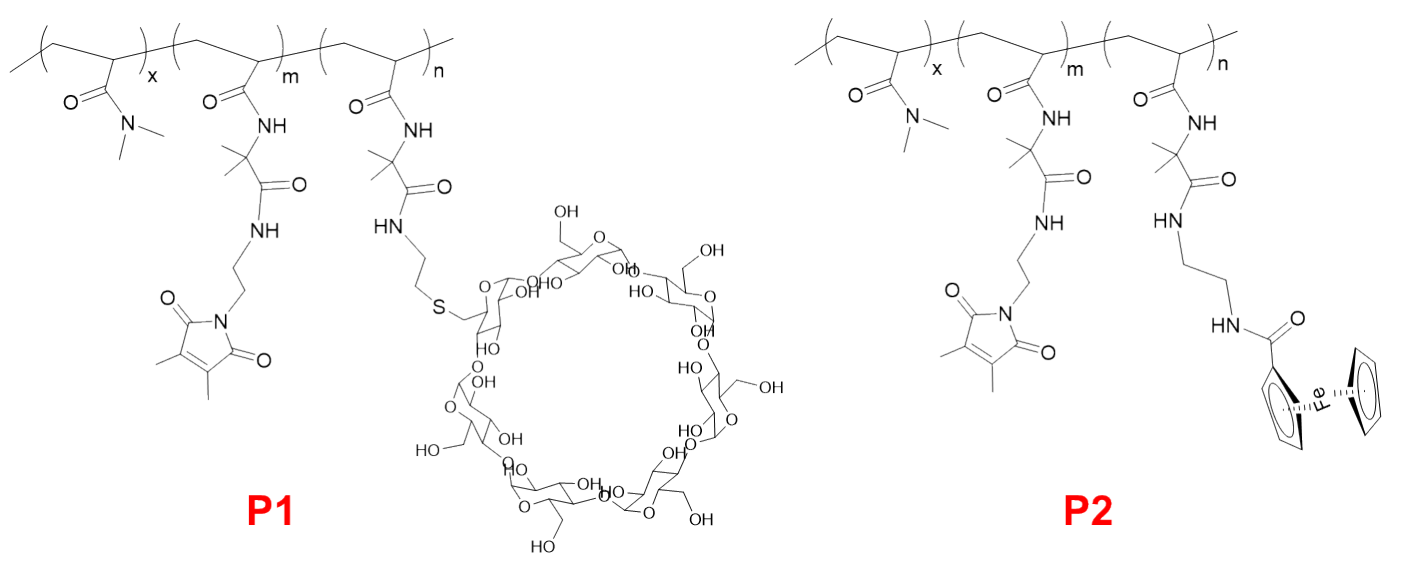

P2

Figure S1. The structures of $\mathbf{P 1}$ and $\mathbf{P 2}$. 


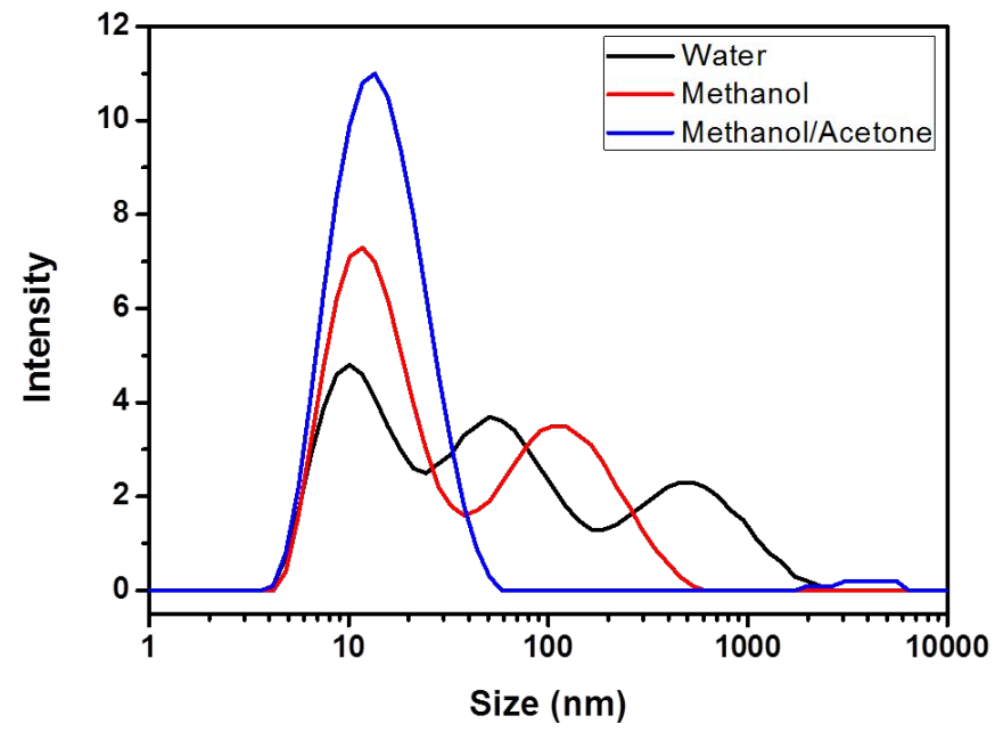

Figure S2. DLS measurements of mixed solution of $\mathbf{P 1}$ and $\mathbf{P} 2$ in different solvents.

a
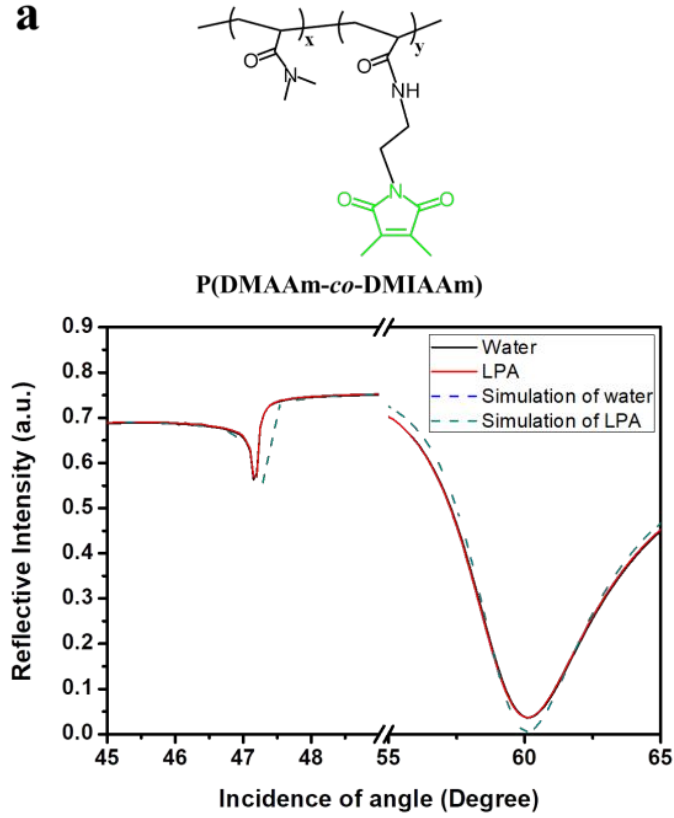

b

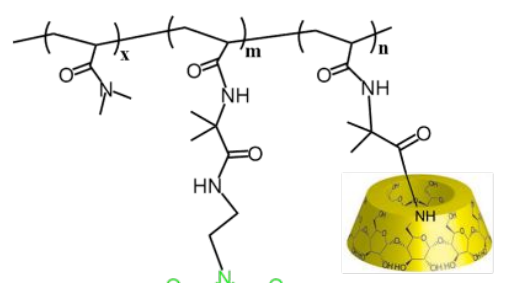

P1

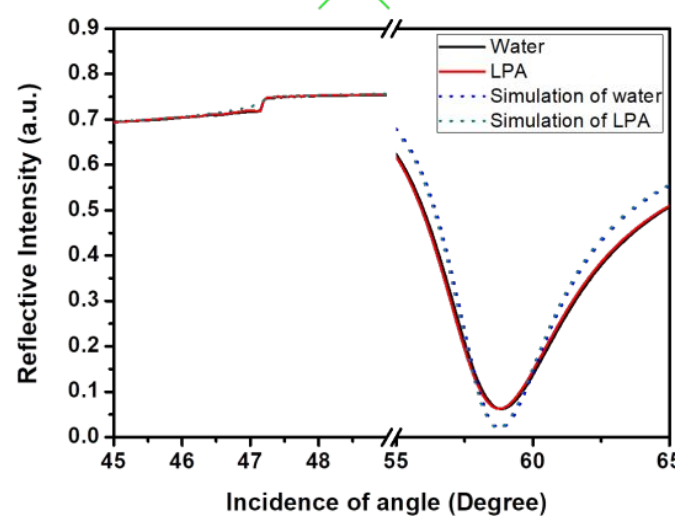

Figure S3. a) Chemical structure of P(DMAAm-co-DMIAAm) and the SPR-OWS measurements upon adding LPA solution $(10 \mu \mathrm{M})$; b) Chemical structure of $\mathbf{P 1}$ and the SPR-OWS measurements upon adding LPA solution $(10 \mu \mathrm{M})$. 
a

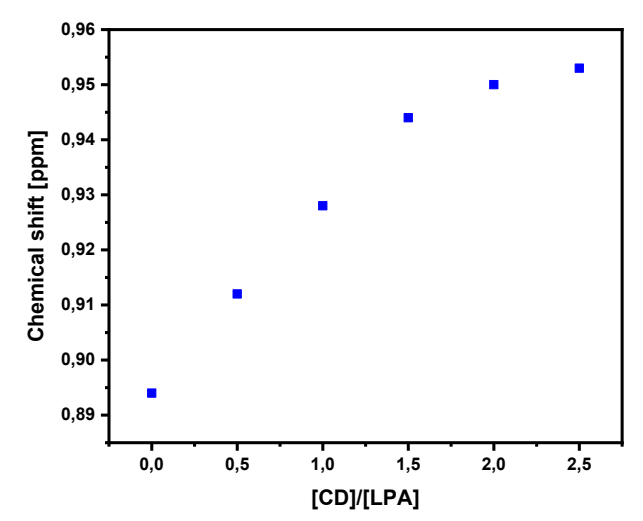

b

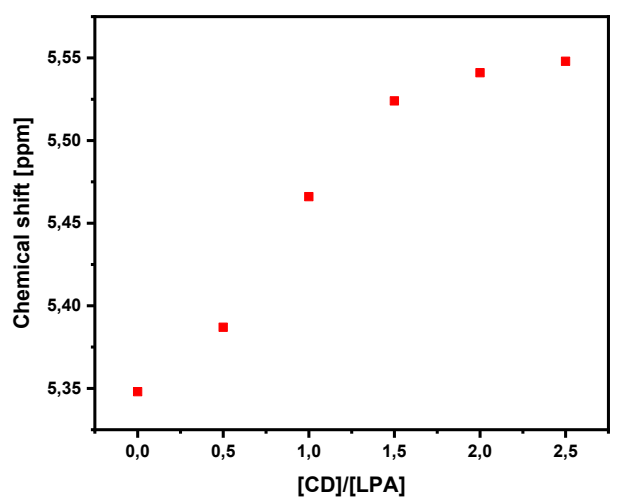

Figure S4. 1H NMR experiments for the LPA and $\beta-C D$ system in $\mathrm{D}_{2} \mathrm{O}$ upon addition of increasing amounts of $\beta$ CD at 298 K. a) Change of chemical shift of LPA protons denoted as $\mathrm{H}_{a}$; b) Change of chemical shift of LPA protons denoted as $\mathrm{H}_{\mathrm{d}}$.
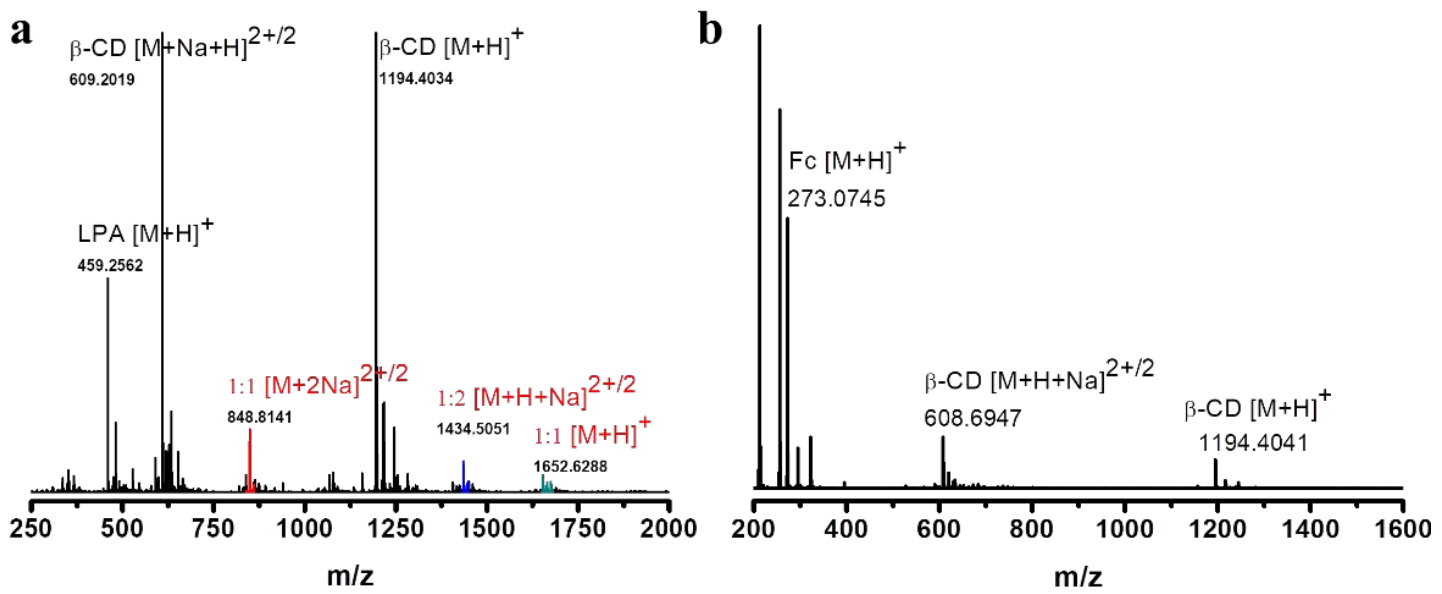

Figure S5. ESI-HRMS for the mixture of host-guest pairs: a) LPA and $\beta$-CD and b) Fc and $\beta$-CD. 


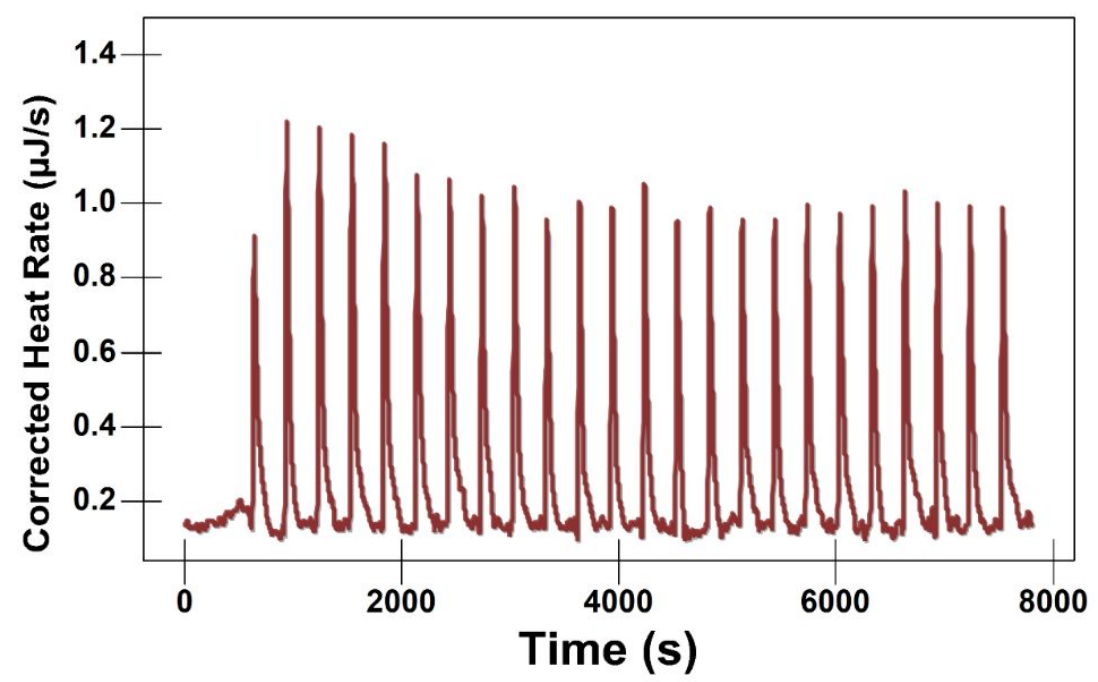

Figure S6. ITC titration curves obtained by injecting LPA solution ( $0.5 \mathrm{mM}$ in Milli-Q water; the volume of each injection: $10 \mu \mathrm{L}$ ) to Milli-Q water at $298.15 \mathrm{~K}$.

a

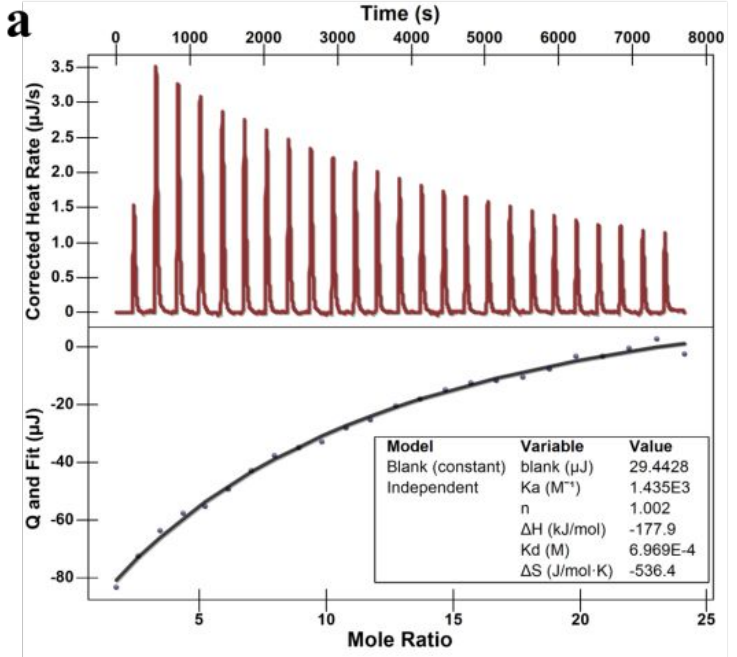

b

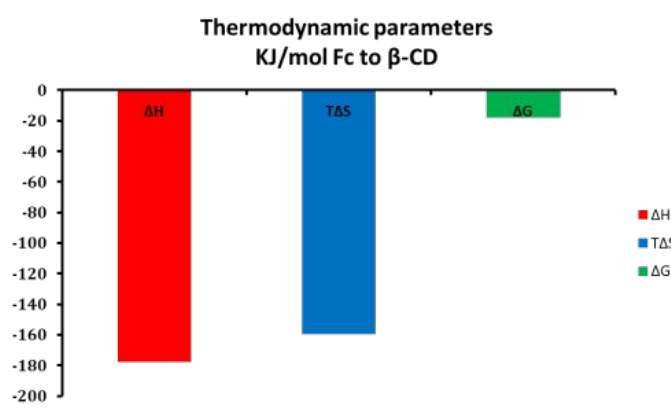

Figure S7. a) Computer simulation of the ITC titration curve obtained by injecting an Ferrocene solution

$(2.50$

$\mathrm{mM}$ in pure water; the volume of each injection: $10 \mu \mathrm{L})$ to a $\beta-\mathrm{CD}$ solution $(0.163 \mathrm{mM}$ in pure water $)$ placed in the reaction cell of ITC instrument at $298.15 \mathrm{~K}$; b) The thermodynamic parameters obtained from the simulation of ITC titration curve: Ferrocene to $\beta-\mathrm{CD}$. 

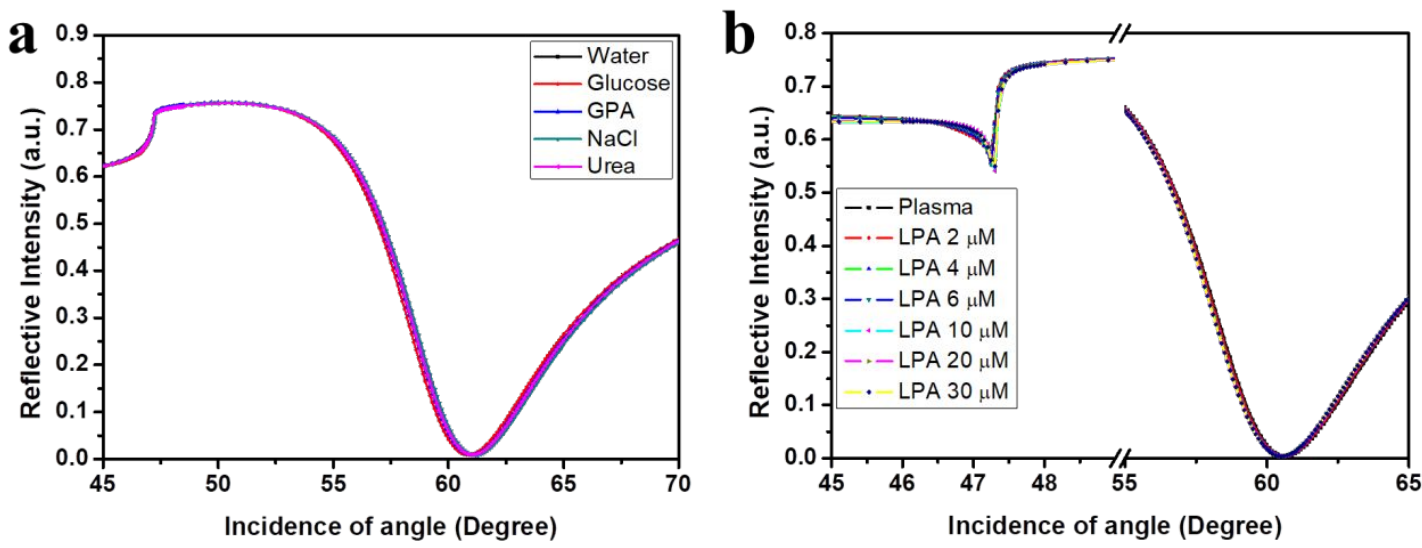

Figure S8. a) SPR-OWS curves of the DCSH with different chemical agents in mimic plasma concentrations:

Glucose (3 mM), GPA $(10 \mu \mathrm{M}), \mathrm{NaCl}(100 \mathrm{mM})$ and Urea $(5 \mathrm{mM})$. b) Concentration dependent measurements of DCSH with different concentrations of LPA in mimic plasma by using SPR-OWS method. The spectrum was break from $49^{\circ}$ to $55^{\circ}$. 
Table S1. Characteristics of the hydrogel film upon its different states.

\begin{tabular}{|c|c|c|}
\hline Materials & Layer Thickness $d_{n}(\mathrm{~nm})$ & Refractive Index $\mathbf{n}_{h}$ \\
\hline Gold layer & 44 & -3.389 \\
\hline Dried Hydrogel & 45 & 1.5942 \\
\hline Hydrogel swelling in water & 734 & 1.3477 \\
\hline Hydrogel swelling in LPA solution & 988 & 1.3461 \\
\hline
\end{tabular}

Table S2. The $\mathrm{pH}$ values of LPA $(50 \mu \mathrm{M}), \beta-\mathrm{CD}(50 \mu \mathrm{M})$, and their mixed solution $(\mathrm{v} / \mathrm{v}=1)$.

\begin{tabular}{cccc}
\hline & LPA & $\beta-C D$ & Mixed solution \\
\hline $\mathrm{pH}$ value & $7.09 \pm 0.05$ & $8.31 \pm 0.05$ & $8.05 \pm 0.09$ \\
\hline
\end{tabular}

Table S3. Calculated energies, thermal and solvent corrections to energies in atomic units.

\begin{tabular}{|c|c|c|c|c|c|c|c|}
\hline Energy in Hartrees & total energy & total thermal corr. & thermal corr. to $\mathrm{H}$ & entropy corr. & solv. corr. & dH & dG \\
\hline $\mathrm{bCD}$ & $-4275,9882$ & 1,3342 & $\quad 0,0009$ & 0,1637 & $-0,0809$ & $-4274,7708$ & $-4274,9232$ \\
\hline bCD-dimer " $(\mathrm{bCD}) 2$ " & $-8551,9882$ & 2,6699 & 0,0009 & 0,2960 & $-0,1335$ & $-8549,5831$ & $-8549,8607$ \\
\hline LPA & $-1692,7367$ & 0,6574 & 0,0009 & 0,0974 & $-0,1216$ & $-1692,1069$ & $-1692,2025$ \\
\hline LPA_bCD-mono1 & $-5968,7501$ & 1,9946 & 0,0009 & 0,2279 & $-0,1815$ & $-5966,9376$ & $-5967,1558$ \\
\hline LPA_bCD-mono2 & $-5968,7440$ & 1,9965 & 0,0009 & 0,2225 & $-0,1474$ & $-5966,9405$ & $-5967,1537$ \\
\hline LPA_(bCD) 2 & $-10244,7933$ & 3,3333 & 0,0009 & 0,3492 & $-0,2019$ & $-10241,8174$ & $-10242,1518$ \\
\hline \multicolumn{8}{|c|}{ dH $[\mathrm{kcal} / \mathrm{mol}] \mathrm{dG}[\mathrm{kcal} / \mathrm{mol}]$} \\
\hline $2 \mathrm{bCD} \rightarrow(\mathrm{bCD}) 2$ & $-26,0189$ & 11,5202 & & & & & \\
\hline$L P A+2 b C D \rightarrow$ LPA_(bCD) 2 & $-105,9498$ & $-7,3506$ & & & & & \\
\hline$L P A+(b C D) 2 \rightarrow L^{-} \_(b C D) 2$ & $-79,9309$ & $-18,8708$ & & & & & \\
\hline $\mathrm{LPA}+\mathrm{bCD}-\mathrm{LPA} \_\mathrm{bCD}-\mathrm{mono1}$ & $-37,5726$ & $-3,5943$ & & & & & \\
\hline LPA + bCD - LPA_bCD-mono2 & $-39,3719$ & 19,4381 & & & & & \\
\hline LPA_bCD-monol + bCD $\rightarrow$ LPA_ & $-68,3772$ & $-3,7563$ & & & & & \\
\hline
\end{tabular}




\section{Experimental section}

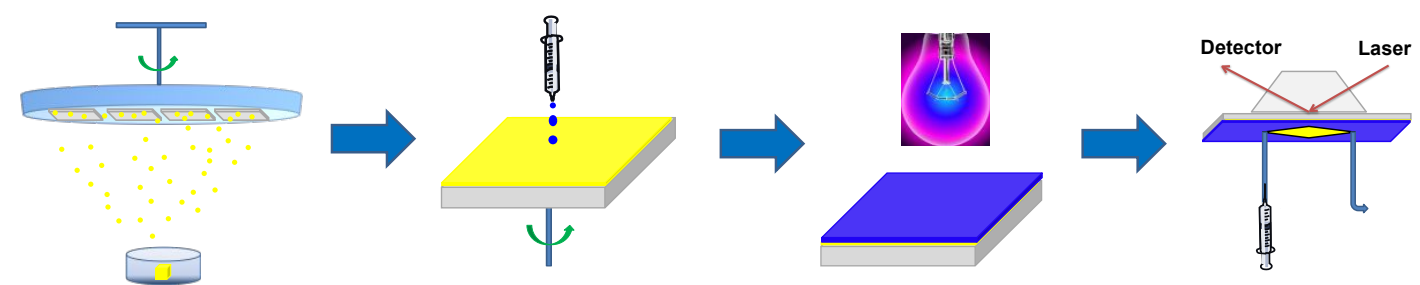

3.1. Gold layer on quartz substrate by PVD:

A size with $2.5 \mathrm{~cm} \times 2.5 \mathrm{~cm}$ of quartz (LaSFN9) was selected as the substrate to prepare the gold surface. Before the quartz was treated with PVD, they should be carefully washed. There were two methods to cleaning the surface of quartz depending on the state of the surface. If the quartz was reused from an old one, they should be cleaned with Piranha solution firstly followed by normal process of cleaning. If the quartz was new, then they just need the normal process of cleaning method as shown below.

Normal process of cleaning quartz surface:

1) ultrasonic cleaning at $55^{\circ} \mathrm{C}$ for $15 \mathrm{~min}$ in water solution with $2 \%$ of Hellmanex ${ }^{\mathrm{TM}} \mathrm{III}$;

2) the quartz was washed distilled water and been ultrasonic cleaning at $55^{\circ} \mathrm{C}$ for $15 \mathrm{~min}$ in triple distilled water for 2 times;

3) the quartz was washed with ethanol and been ultrasonic cleaning at $55^{\circ} \mathrm{C}$ for $15 \mathrm{~min}$ in ethanol for 2 times;

4) the quartz was washed with absolute ethanol, dried with gas gun.

The layer was then treated with PVD for covering a golden surface with around $50 \mathrm{~nm}$ layer thickness. This process was started when the vacuum was under $1 \mathrm{E}-5$ and was stopped when the monitor was shown a layer thickness at $70 \mathrm{~nm}$. The last, SPR measurement was used to check the layer thickness of the gold layer.

\subsection{Spin coating of polymer layer on gold surface}

\section{Preparation of gold surface:}

The gold surface was treated with ethanol solution of additional promotor, which can act as an anchor to connect gold surface with dually crosslinked supramolecular gel. The concentration of additional promotor was $5 \mathrm{mM}(1.2 \mathrm{mg} / \mathrm{mL})$. The gold substrate was submerged in additional 
promotor solution for 12 hours followed by washing with ethanol and dried with gas gun.

\section{Preparation of polymer solution:}

Science the viscosity of polymer solution has great influence in the layer thickness of polymer thin layer, concentration of polymer and type of solvent were selected carefully. For the hydrophobic polymer, chloroform was carried out as a good solvent. For the hydrophilic polymer, methanol was used as a good solvent. Different concentrations of polymer were checked using the same spin coating method. $10 \mathrm{mg} / \mathrm{mL}$ was thought the superior concentration for polymer thin layer under 100 $\mathrm{nm}$ in day state. The polymer solution was stirring for 12 hours at room temperature to ensure a complete dissolution of the polymer. After that, the solution was through a filter $(0.45 \mu \mathrm{m})$ before spin coating.

\section{Parameter setting of the spin coater:}

Parameters of spin coater could be influence in the quality of polymer layer, like homogeneous and layer thickness. Considering of the properties of solvents we used here, the recipe of the spin coater was set as: 1) $250 \mathrm{rmp}$ for 25 seconds; 2) $2500 \mathrm{rmp}$ for 70 second; 3) $0 \mathrm{rmp}$ for 2 seconds.

\subsection{Photo crosslinking of dually crosslinked supramolecular gel}

After the polymer layer was spin coating, the slice was dried overnight at room temperature in dark. Then they were prepared to photo crosslink by using a UV-Vis lamp. Parameters of the UV-Vis lamp were shown as below. The distance between lamp and sample was $8 \mathrm{~cm}$; the light intensity on the sample is $252 \mathrm{~mW} / \mathrm{cm}^{2}$ ( $43 \%$ of the intensity from the monitor); irradiation time of the sample is 65 second.

\subsection{Layer thickness detection by SPR/HOWS}

SPR/HOWS spectra were recorded in the Kretschmann configuration with a customized setup from Max Planck Institute for Polymer Research (Mainz, Germany). The SPR/HOWS sensor was implemented by using the optical setup described in Figure S9. Briefly, A monochromatic light (He/Ne laser, $\lambda=632.8 \mathrm{~nm}$, Uniphase) was passed through polarizers (Glan-Thompson polarizer, B. Halle) to obtain linear and transverse magnetic (TM, p) polarization and was reached to a high refractive index prism (LASFN9, refractive index $n_{p}=1.8449$ ). The sensor chip was covered by a flow cell through a rubber gasket $\left(1 \mathrm{~cm}^{2} \times 0.5 \mathrm{~cm}\right)$, which selected an area around $1 \mathrm{~cm}^{2}$ from the 
sensor chip as the efficient area for the next responsive measurement. The prism moiety and the sensor chip were connected by using an immersion oil (Cargille Lab., USA, refractive index $n 25^{\circ} \mathrm{C}$ $\mathrm{D}=1.8000 \pm 0.005$ ), which has the similar refractive index with the prism. Then, this pack was mounted on a rotation stage (2-circle 414 with the precision $10^{-2}$ deg, Huber AG, Germany) to control the angle of incidence from the laser beam $\theta$. The reflected intensity of the laser beam after coupled with the surface plasmon of gold was measured by using a photodiode connected to a lockin amplifier (7265 DSP Lock-in amplifier, Signal Recovery, USA, integration time 3 S).

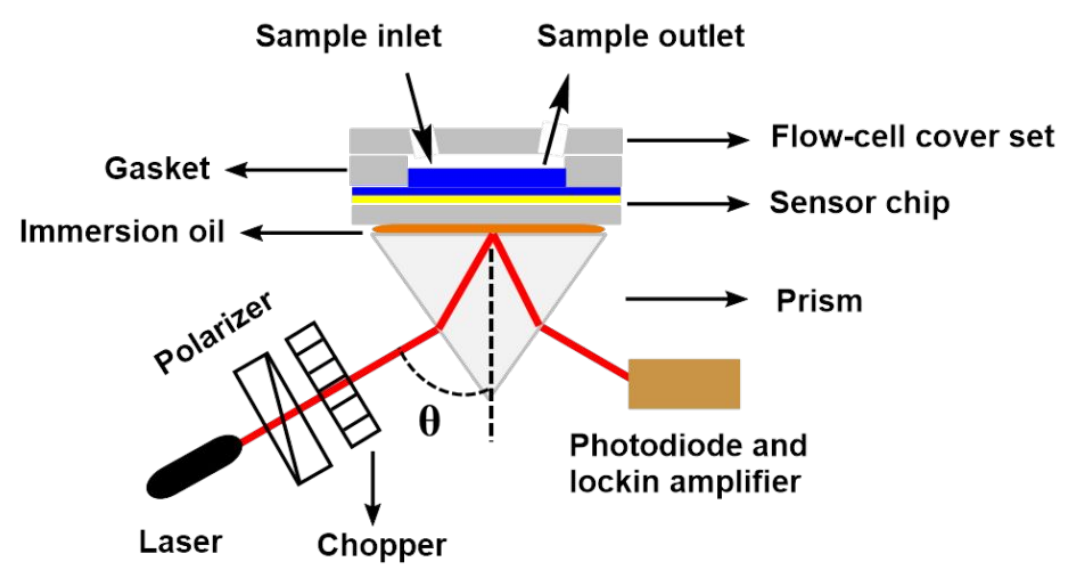

Figure S9. The scheme of optical setup and sensor chip for the excitation of optical waveguide (OW) and surface plasmon (SP).

The excitation of SP/OW waves from the laser beam hitting the gold layer and causing resonance with surface plasmon of gold layer was observed from the angular dependent reflectivity spectra $R(\theta)$. This spectrum was manifested as two distinct dips that belong to layer thickness $\left(d_{h}\right)$ and refractive index $\left(n_{h}\right)$, respectively. In order to determine the $d_{h}$ and $n_{h}$, the angular reflectivity spectrum exhibiting SP and HW resonance dips was fitted by transfer matrix-based model through a software Winspall (MPI polymer research in Mainz, Germany).

[1] S. Grimme, C. Bannwarth, P. Shushkov, J. Comput. Theory Chem. 2017, 13, 1989-2009.

[2] Neese, F. "The ORCA program system" Wiley Interdiscip. Rev. Comput. Mol. Sci. 2012, 2, 73 78.

[3] a) H.Kruse, S. Grimme J. Chem. Phys., 2012, 136, 154101; b) S. Grimme, J. Antony, S. Ehrlich, H. Krieg, J. Chem. Phys. 2010, 132, 154104; c) S. Grimme, J. G. Brandenburg, C. Bannwarth, A. 
Hansen J. Chem. Phys. 2015, 143, 054107; d) F. Weigend, Phys. Chem. Chem. Phys. 2006, 8, 1057. [4] a) S. Grimme, S. Ehrlich, L. Goerigk, J. Comput. Chem. 2011, 32, 1456-1465; b) S. Grimme, J. Antony, S. Ehrlich, H. Krieg, J. Chem. Phys. 2010, 132, 154104.

[5] a) F. Weigend, R. Ahlrichs, Phys. Chem. Chem. Phys. 2005, 7, 3297; b) F. Weigend, Phys. Chem. Chem. Phys. 2006, 8, 1057. 